\title{
Inkrementelle Kosten der A/H1N1-Pandemie für den Öffentlichen Gesundheitsdienst - Berechnungen am Beispiel der Metropole Frankfurt am Main
}

\author{
Costs of the Influenza Pandemic for the Public Health Services - Calculations \\ Based on the Model of the Metropolitan Region Frankfurt am Main
}

Autoren

Institute
R. Gottschalk ${ }^{1,2}$, O. Bellinger ${ }^{1}$, S. Stadthagen ${ }^{1}$, U. Götsch ${ }^{1}$, M. Creß ${ }^{1}$, R. Diel ${ }^{3}$

Amt für Gesundheit Stadt Frankfurt am Main

Institut für Medizinische Virologie, Klinikum und Fachbereich Medizin der Johann Wolfgang Goethe-Universität Frankfurt am Main

Abteilung für Pneumologie, Medizinische Hochschule Hannover

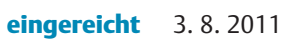
akzeptiert nach Revision 9. 9.2011

\section{Bibliografie}

Dol http://dx.doi.org/ 10.1055/s-0031-1291394 Online-Publikation: 2.11.2011 Pneumologie 2011; 65: 697-704 (c) Georg Thieme Verlag KG Stuttgart · New York ISSN 0934-8387

Korrespondenzadresse Prof. Dr. Dr. René Gottschalk Amt für Gesundheit Stadt Frankfurt am Main Breite Gasse 28 60313 Frankfurt am Main rene.gottschalk@stadt-frankfurt. de

\section{Zusammenfassung \\ $\nabla$}

Eine der größten Herausforderungen für den Öffentlichen Gesundheitsdienst (ÖGD) der deutschen Nachkriegsgeschichte stellte die A/H1N1Pandemie des Jahres 2009 dar. Die entsprechend dem Infektionsschutzgesetz (IfSG) privilegierte Einbindung des ÖGD macht eine methodisch transparente Erörterung der hierdurch verursachten Gesamtkosten erforderlich. In der folgenden Analyse wurden daher exemplarisch für die Metropole Frankfurt am Main alle relevanten Kostenblöcke für das Kalenderjahr 2009 erfasst, welche die Kosten der Neuen Grippe widerspiegeln können. Die inkrementellen Präventionsund Logistikkosten, die dem Amt für Gesundheit Frankfurt am Main zur Bewältigung der H1N1Pandemie, einschließlich der Implementierung eines modernen Serversystems zur Impfplanung und -koordination entstanden, betrugen für das Jahr $2009 € 223537,91$. Die direkten Impfkosten (ohne Impfstoff) betrugen stattdessen lediglich $€ 45401,48$, d.h. bei 4261 durchgeführten Impfungen nur $€ 10,66$ pro geimpftem Bürger. Da mit Pandemien auch künftig gerechnet werden muss, sollte auch im Hinblick auf zukünftige Rationalisierungs- und Reallokationsbestrebungen für eine ausreichende finanzielle Ausstattung des ÖGD gesorgt werden.

\section{Einleitung}

$\nabla$

Am 25.4.2009 deklarierte die Weltgesundheitsorganisation (WHO) die Ausbreitung des A/ H1N1-Virus offiziell als ein ,internationales Ereignis mit Bedrohung der öffentlichen Gesundheit“. Sechs Jahre nach der SARS-Epidemie war dies die zweite Pandemie des 21. Jahrhunderts. Für Frankfurt am Main und die Rhein-Main-Region stellte die Pandemie der „Neuen Grippe“ eine besondere Herausforderung dar, da der RheinMain-Flughafen mit knapp 51 Mio. Passagieren

\section{Abstract \\ $\nabla$}

The influenza pandemic of 2009 has been the biggest challenge to the public health services in post-war Germany. This study investigates the impact on the overall costs for the public health authorities of the metropolitan region Frankfurt am Main which arose in the context of the pandemic as well as the specific costs of the implementation and realisation of the vaccination campaign during the pandemic. In 2009 the incremental costs for the Health Protection Authority of the City of Frankfurt am Main for the prevention and logistics caused by this pandemic amounted to $€ 223,537.91$, whereas costs which could be directly attributed to the vaccination campaign (vaccine not included amounted to only a fraction thereof ( $€ 45,401.48$ ). The per-capita costs for vaccinated citizens were $€ 10.66$. These results clearly demonstrate the importance of adequate financial resources for the public health authorities to cope with infectious disease outbreaks and future pandemics.

(2009) den neunten Platz im weltweiten Passagieraufkommen belegt. Hinzu kommt, dass -im Gegensatz zu anderen deutschen Großstädten eine direkte Verbindung zu der zuerst betroffenen Region (Mexiko) mit 10 Non-Stop-Flügen und damit fast 3500 Passagieren pro Woche besteht. Durch die darüber hinaus vor allem in den USA rasch zunehmenden Fallzahlen rückte der Flughafen noch weiter in den Mittelpunkt, weil in Frankfurt aus den USA durchschnittlich 47 Maschinen mit insgesamt ca. 13000 Passagieren täglich landen. Deshalb wurde der Schwerpunkt des 
Seuchenmanagements bei der Neuen Influenza A/H1N1 zunächst auf den Flughafen im Sinne eines „Entry-Screenings“ gelegt [1].

Das Problemfeld „Flughafen“ trat jedoch rasch in den Hintergrund, da die autochthonen (innerdeutschen) Übertragungsfälle sehr schnell zunahmen und eine Verhinderung der Einschleppung neuer Fälle durch Entry-Screening unsinnig wurde. Angesichts der damaligen Modellannahmen musste man davon ausgehen, dass sich etwa 30\% der deutschen Bevölkerung mit dem H1N1-Virus infizieren würden, wobei alleine in Frankfurt am Main von 50000-140000 Personen auszugehen war [2]. Von entscheidender Bedeutung wurde daher die künftige Impfung mit dem zum damaligen Zeitpunkt noch in der Erprobung befindlichen Impfstoff erachtet.

Ungeachtet dessen war seit Auftreten der ersten Fälle in Deutschland der Informationsbedarf unter der Bevölkerung über Krankheitsprophylaxe und -verlauf, später auch über Wirksamkeit und mögliche Risiken des neuen Impfstoffes, verbunden mit dem Wunsch nach individueller telefonischer Beratung, außerordentlich hoch.

Allen drei Herausforderungen, d.h. den Containment-Bemühungen am Flughafen, der raschen Zunahme innerdeutscher Übertragungen und der hieraus resultierenden Notwendigkeit eines Impfangebotes, musste - in unterschiedlichen zeitlichen und sich z.T. überschneidenden Abfolgen - mit adäquaten Konzepten durch den ÖGD begegnet werden. Diese und die hieraus resultierenden Kosten für den Frankfurter Öffentlichen Gesundheitsdienst, repräsentiert durch das Amt für Gesundheit, werden nachfolgend für die Metropole Frankfurt am Main mit ihrer besonderen Flughafenanbindung en detail dargestellt.

\section{Methodik}

$\nabla$

Diese Analyse erfolgt ausschließlich aus der Perspektive des Öffentlichen Gesundheitsdienstes der Stadt Frankfurt am Main und umfasst daher lediglich die im Rahmen der H1N1-Pandemie anfallenden zusätzlichen Kosten des Amtes für Gesundheit (AfG). Diese setzen sich aus Personal- und Sachkosten zusammen.

Entstandene Sachkosten werden, mittels doppelter Buchführung den einzelnen Titeln zugeordnet, durch den Vergleich der Aufwendungen und Erträge des aktuellen Jahres in der Kosten- und Leistungsrechnung angegeben. Dauerhafte Anschaffungen wurden summarisch bzw. bezogen auf das Pandemiejahr 2009 unter Berücksichtigung möglicher Abschreibungen gemäß $\S 6$ Abs.2a Einkommenssteuergesetz (EStG) dargestellt.

Für die Berechnung der Kosten für Personal wurde daher auf die Allgemeine Verwaltungskostenordnung (AllgVwKostO) des Landes Hessen vom 21.11.2003 (17 - Gesetz- und Verordnungsblatt für das Land Hessen, Teil I - 27.11.2003) zurückgegriffen. Hiernach können für noch näher zu spezifizierende Amtshandlungen Gebühren nach summarischem Zeitaufwand der beteiligten Beschäftigten erhoben werden. Gestaffelt nach höherem, gehobenem und übrigem Beamtendienst bzw. vergleichbaren Angestellten-Tätigkeiten werden je begonnener $1 / 4$ Stunde $€ 18,-$, $€ 15,-$ oder $€ 12,25$ berechnet (Nr.1411-1413), d.h. €72,-, €60,- und $€ 49$,- pro Stunde. Nachfolgend werden die Kosten für ärztliche Tätigkeit dem höheren, für Verwaltungstätigkeiten dem gehobenen und für alle sonstigen Tätigkeiten durch Mitarbeiter/-innen des AfG dem „übrigen“ Dienst zugeordnet.
Im Rahmen der Aufgabenerfüllung für die Pandemieschutzmaßnahmen entstandene Personalkosten pro Stunde wurden vereinfachend nur dann in die Kostenkalkulation einbezogen, wenn sie zu dokumentierten Überstunden führten oder die Mitarbeiter/-innen durch exklusive Mitarbeit am Bürgertelefon bzw. Impfanmeldung im Zeitraum vom 29.4.2009 - 8. 5. 2009 („Mexikophase“) und 22.6.2009-31.12.2009 („Deutschlandphase“) von ihrer regulären Arbeitstätigkeit ausgeschlossen waren.

\section{Maßnahmen und deren Kosten}

$\nabla$

\section{Eindämmungsphase („Mexikophase“)}

Ausbreitungskontrolle am Flughafen

Ein ärztlicher und ein nichtärztlicher Mitarbeiter der Flughafenklinik Frankfurt und bei Bedarf ein Arzt des AfG kontrollierten seit dem Morgen des 29.4.2009 in allen Maschinen aus Mexiko und weiteren Flugzeugen mit H1N1-Verdachtsfällen die Passagiere. Die Passagiere wurden per Durchsage informiert und um Geduld und Mithilfe gebeten. Zunächst wurde die Crew, danach die Reisenden Reihe für Reihe nach grippeähnlichen Symptomen befragt und angeschaut. Ergaben sich keine Auffälligkeiten wurde das Flugzeug wieder freigegeben.

Befand sich an Bord ein mit der „Neuen Grippe“ vereinbarer Verdachtsfall, wurde der Patient direkt in die Isolierstation der Frankfurter Uni-Klinik gebracht. Beim Verlassen des Flugzeugs mussten die Mitreisenden sog. „Aussteigerkarten“ mit ihren persönlichen Daten inkl. ihrer Mobilfunknummer ausfüllen. Bestätigte nach einer Latenz von etwa sechs Stunden ein PCR-Schnelltest den H1N1-Verdacht, wurden die anderen Passagiere vom Amt für Gesundheit kontaktiert. Da infizierte, aber noch nicht symptomatische Passagiere auch erst Tage nach ihrer Ankunft Symptome entwickeln konnten, verteilten die Mitarbeiter, welche auch die Maschinen begingen, Informationsmaterialien.

Ab dem 7.5.2009 wurden aufgrund der zunehmenden Inlandsübertragungen keine Aussteigerkarten mehr ausgefüllt und am Flughafen lediglich weiter Informationsmaterialien ausgeteilt. Dieses wurde mit dem Ende der Containment-Phase Anfang Juni 2009 wieder eingestellt, die Poster wurden Anfang 2010 vom Terminalbereich des Flughafens entfernt.

Während dieser „Mexikophase“ wurden durch das Amt für Gesundheit $€ 26838$,66 aufgewendet (s. Tab.1, Zeile 46). Prophylaktische Schutzmaßnahmen für die Mitarbeiter für Mundschutz (€2644,94, enthalten in Zeile 10, ebd.) und Händedesinfektionsmittel (€2055,00, enthalten in Zeile 10, ebd.) machten dabei lediglich $€ 4699,94$ aus. Das Gros entfiel allerdings mit $€ 16601,21$ auf Aufwendungen für Öffentlichkeitsarbeit (€10990,36 für InfoBlätter, enthalten in Zeile 33, ebd., €4040,05 für Aussteigerkarten, enthalten in Zeile 33, ebd., sowie €1570,80 für Plakate, enthalten in Zeile 33, ebd.).

\section{Aufbau einer Telefon-Hotline - Bürgertelefon}

Mit dem Beginn der „Mexikophase“ und dem Import reiseassoziierter H1N1-Erkrankungen wurde ab dem 29.4.2009, zeitgleich mit 23 anderen Gesundheitsämtern in Hessen, eine „Hotline“ geschaltet, die für besorgte Bürger wochentags von 8:00 bis 17:00 Uhr erreichbar war. Dieses separate Bürgertelefon wurde in Frankfurt am Main zeitgleich mit bis zu 5 Personen nicht-ärztlichen, medizinischen Personals besetzt. 
Tab. 1 Sachkosten der Neue Grippe-Pandemie für das Amt für Gesundheit Frankfurt am Main 2009 (dauerhafte Anschaffungen kursiv).

\begin{tabular}{|c|c|c|c|c|}
\hline \multirow[t]{2}{*}{ Bezeichnung Sachkonto } & Bezeichnung Aufwand/Ertrag & Datum & Gesamt & Gesamt \\
\hline & & 2009 & $\begin{array}{l}\text { "Mexiko- } \\
\text { phase“ }\end{array}$ & $\begin{array}{l}\text { "Deutsch- } \\
\text { landphase“ }\end{array}$ \\
\hline \multirow[t]{2}{*}{ Rohstoffe/Material } & Schutzkappen für Fieberthermometer & $05 / 2009$ & $494,48 €$ & $0,00 €$ \\
\hline & med. Sachbedarf, sonstiges & $05+11 / 2009$ & $138,71 €$ & $733,40 €$ \\
\hline \multirow{3}{*}{$\begin{array}{l}\text { Aufwendungen für Büromaterial und } \\
\text { Drucksachen der Verwaltung und } \\
\text { ähnl. Einrichtungen }\end{array}$} & diverse IT-Materialien, sonstiges & $09 / 2009$ & $0,00 €$ & $536,10 €$ \\
\hline & & $11 / 2009$ & & \\
\hline & Kartei-Leitregister, Karteikästen & $05 / 2009$ & $180,42 €$ & $0,00 €$ \\
\hline $\begin{array}{l}\text { Materialaufwand für Einrichtungen und } \\
\text { Ausstattungen }\end{array}$ & Beistelltische & $05+11 / 2009$ & $0,00 €$ & $131,64 €$ \\
\hline Materialaufwand für Kfz & Magnethaftschilder & $06 / 2009$ & $419,00 €$ & $0,00 €$ \\
\hline $\begin{array}{l}\text { Aufwendungen für Berufskleidung, } \\
\text { Arbeitsschutzmittel }\end{array}$ & Fleecejacken, Strickhandschuhe, Arztkittel & $10+11 / 2009$ & $0,00 €$ & $370,41 €$ \\
\hline \multirow[t]{5}{*}{ Reinigungsmaterial } & $\begin{array}{l}\text { Einmaltaschentücher, Mundschutz, Hände- } \\
\text { desinfektionsmittel }\end{array}$ & $\begin{array}{l}08+09 / 2009 \\
11 / 2009\end{array}$ & $4963,05 €$ & $163,63 €$ \\
\hline & Messkolben & $10 / 2009$ & $0,00 €$ & $8,57 €$ \\
\hline & $\begin{array}{l}\text { Einweghandschuhe, Schutzhandschuhe, Pumpaufsatz } \\
\text { für Sterilium-Flasche }\end{array}$ & $10+11 / 2009$ & $0,00 €$ & $1037,03 €$ \\
\hline & Bode-X-Wipes-Eimer rot & $10 / 2009$ & $0,00 €$ & $416,20 €$ \\
\hline & $\begin{array}{l}\text { Bode-X-Wipes, Vliesrolle Nachfüllpackung, Kohrsolin } \\
\text { (5-I-Kanister), sonstiges }\end{array}$ & $11 / 2009$ & $0,00 €$ & $1573,49 €$ \\
\hline \multirow[t]{3}{*}{ übriger sonstiger Materialaufwand } & Transportwagen, Kühltasche, Ice-Akku & $10 / 2009$ & $0,00 €$ & $213,31 €$ \\
\hline & Laptoptasche & 06/2009 & $62,95 €$ & $0,00 €$ \\
\hline & DKT-Taschen, neutral weiß (Plastiktüten), sonstiges & $10+12 / 2009$ & $0,00 €$ & $118,39 €$ \\
\hline sonstige Fremdinstandhaltung, Wartung & Materialaufwand technischer Anlagen (ISS Serversensor) & $12 / 2009$ & $0,00 €$ & $1335,50 €$ \\
\hline Fremdreinigung & WISAG (Abruf einer Hilfskraft) & & $0,00 €$ & $720,14 €$ \\
\hline $\begin{array}{l}\text { andere sonstige Aufwendungen für } \\
\text { bezogene Leistungen }\end{array}$ & Sonstiges & $08 / 2009$ & $0,00 €$ & $116,98 €$ \\
\hline übrige sonstige Personalaufwendungen & Stornierungskosten Urlaub & $10 / 2009$ & $0,00 €$ & $940,00 €$ \\
\hline \multirow{11}{*}{$\begin{array}{l}\text { sonstige Aufwendungen für die } \\
\text { Inanspruchnahme von Diensten }\end{array}$} & Transport von Impfstoff & $10 / 2009$ & $0,00 €$ & $1273,30 €$ \\
\hline & 10 Schlüsseltransponder & $10 / 2009$ & $0,00 €$ & $623,32 €$ \\
\hline & Installation Workstations für Pandemie & $09 / 2009$ & $0,00 €$ & $1142,40 €$ \\
\hline & Unterstützung bei der Installation & $12 / 2009$ & $0,00 €$ & $2337,46 €$ \\
\hline & $\begin{array}{l}\text { Transport Pandemiekeller Braubachstr. nach } \\
\text { Breite Gasse }\end{array}$ & $05 / 2009$ & $397,67 €$ & $0,00 €$ \\
\hline & Transport von mobilen Stellwänden & $05 / 2009$ & $108,46 €$ & $0,00 €$ \\
\hline & Dienstleistung/Techniker & $05 / 2009$ & $324,87 €$ & $0,00 €$ \\
\hline & Mikrofon- und Beschallungstechnik & $05 / 2009$ & $687,82 €$ & $0,00 €$ \\
\hline & Arbeitsübersetzung* & $05 / 2009$ & $331,77 €$ & $0,00 €$ \\
\hline & Rücktransport von mobilen Stellwänden & $07 / 2009$ & $188,92 €$ & $0,00 €$ \\
\hline & Sonstiges & $05 / 2009$ & $894,00 €$ & \\
\hline \multirow[t]{2}{*}{ Aufwendungen für Öffentlichkeitsarbeit } & $\begin{array}{l}\text { Aussteigerkarten, Arbeitsübersetzung inkl. Eilzuschläge, } \\
\text { Plakate, Infoblätter, Druckkosten* }\end{array}$ & $\begin{array}{l}05+06 / 2009 \\
09+11 / 2009\end{array}$ & $17210,85 €$ & $2054,36 €$ \\
\hline & Beschallung für eine Pressekonferenz & $08 / 2009$ & $359,74 €$ & $0,00 €$ \\
\hline Aufwendungen für Gästebewirtung & Getränke & $11 / 2009$ & $0,00 €$ & $86,34 €$ \\
\hline $\begin{array}{l}\text { Aufwendungen für Schadenersatz- } \\
\text { leistungen }\end{array}$ & Sonstiges & 08/2009 & $0,00 €$ & $199,43 €$ \\
\hline übrige sonstige betriebliche Aufwendungen & Sonstiges & $05 / 2009$ & $75,95 €$ & $0,00 €$ \\
\hline $\begin{array}{l}\text { Erwerb von beweglichem Vermögen } \\
\text { (Sammelanlagen) GWG }\end{array}$ & $\begin{array}{l}\text { Wandsystem (Trennwände wegen Akustik in } \\
\text { Call-Centern), Abdeckband }\end{array}$ & & $0,00 €$ & $7610,04 €$ \\
\hline \multirow[t]{2}{*}{ Erwerb von beweglichem Vermögen } & Latitude, N-Service Intel Core (9 Laptops) & $11 / 2009$ & $0,00 €$ & $11781,00 €$ \\
\hline & $\begin{array}{l}2 \text { Server }(9313,33 €) \text {, } 4 \text { Jahre Vor-Ort-Service } \\
(986,65 €=246,66 € \text { anteilig für } 1 \text { Jahr })\end{array}$ & $11 / 2009$ & $0,00 €$ & $9559,99 €$ \\
\hline \multirow[t]{7}{*}{ Erwerb imm. Vermögensgegenstände } & Erstellung einer Impfdatenbank & $12 / 2009$ & $0,00 €$ & $18649,68 €$ \\
\hline & Installation eines Webservers & $11 / 2009$ & $0,00 €$ & $2378,57 €$ \\
\hline & Schulung der Impfstellen & $12 / 2009$ & $0,00 €$ & $4473,45 €$ \\
\hline & Beratungsleistungen für Vorarbeiten & $11 / 2009$ & $0,00 €$ & $1713,60 €$ \\
\hline & $\begin{array}{l}\text { laufende Betreuung, Wartung und Erweiterung } \\
\text { Impfdatenbank }(10-12 / 2009)\end{array}$ & $11 / 2009$ & $0,00 €$ & $4688,12 €$ \\
\hline & Summen & Gesamt & $26838,66 €$ & $76985,85 €$ \\
\hline & hiervon dauerhaft Anschaffungen & & $1156,85 €$ & $53456,33 €$ \\
\hline
\end{tabular}

${ }^{*}$ Diese Aufwendungen sind Flughafenkosten. 


\begin{tabular}{|lrrr|}
\hline 29.4.2009 bis 8.5.2009 („Mexikophase“) & & \\
& Stunden & Kosten je Stunde & Kosten gesamt \\
\hline PK* Bürgertelefon & 212,00 & $49,00 €$ & $10388,00 €$ \\
\hline Überstunden ärztl. Personal & 106,05 & $72,00 €$ & $7635,60 €$ \\
\hline Überstunden Personal Verwaltung & 51,89 & $60,00 €$ & $3113,40 €$ \\
\hline PK* gesamt & 369,94 & & $21137,00 €$ \\
\hline
\end{tabular}

Tab. 2 Personalkosten der Hotline für die „Mexikophase“ der H1N1-Pandemie 2009.
Nach dem 8.5.2009 wurde mit dem Abflauen der Nachfrage die Hotline wieder eingestellt; die (vereinzelten) Anfragen wurden wieder in den „normalen“ Dienstbetrieb der Gesundheitsaufseher überführt und monetär nicht mehr gesondert ausgewiesen. Überstunden der Verwaltung entstanden für die Planung und Logistik des aufsuchenden Szenarios am Flughafen bzw. der Hotline. Für diese erste Hotline der H1N1-Pandemie entfielen für das AfG Frankfurt an Personalkosten somit insgesamt €21137 (s. - Tab. 2).

\section{Impfphase („Deutschlandphase“)} Impfkoordination

Ab dem 26. 10.2009 wurde in Deutschland bundesweit nach Absprache der Ländergesundheitsminister mit den Impfungen gegen die Neue Influenza A/H1N1 begonnen. Zur Koordination und Dokumentation der Impfungen in der Stadt Frankfurt am Main wurde hierfür, durch das AfG, in Zusammenarbeit mit der Firma „mainis IT-Service GmbH“ eine internetbasierte SoftwareAnwendung erstellt. Der Impfstoff wurde in Frankfurt zentral dem AfG angeliefert, dort verwahrt und an die kooperierenden Betriebsärzte der bis zu 30 Impfstellen, nämlich die Impfstelle des Amtes für Gesundheit (bis zu 12 Impfbereiche), 16 Frankfurter Krankenhäuser, Impfstellen öffentlicher Einrichtungen (z.B. Feuerwachen) und einzelner Großunternehmen übersandt.

Die Anwendung wurde auf einem Internetserver der Stadt Frankfurt am Main zur Verfügung gestellt und administriert, sodass von allen Impfstellen mittels Internetzugang über einen gewöhnlichen Webbrowser online darauf zugegriffen werden konnte.

Ziel des Programms war es, möglichst viele Impfungen mit dem geringstmöglichen Zeit- und Personalaufwand durchzuführen und den Verbleib des Impfstoffes nachzuverfolgen. Der Server erlaubte dabei den eingerichteten Impfstellen, Impfungen online zu erfassen bzw. durch die Vergabe von Impfterminen vorab zu planen und vor Ort zu koordinieren.

Durch einen geeigneten Zugriffsschutz in Verbindung mit einem abgestuften Berechtigungskonzept wurde sichergestellt, dass die Impfdaten nur durch die jeweils berechtigten Personen eingesehen werden konnten. Alternativ bestand die Möglichkeit, die vor Ort in einem Tabellenblatt erfassten oder aus einer Verwaltungssoftware exportierten Impfdaten an zentraler Stelle in die Anwendung zu importieren.

Es wurden dabei unter Berücksichtigung des Datenschutzes nur Daten erhoben und gespeichert, die zur Durchführung und Dokumentation der Impfung notwendig waren: Personendaten, Daten zur Impfung, in geringerem Umfang auch medizinische Daten.

Im Vorfeld der Impfungen konnten über ein bei dem Amt für Gesundheit eingerichtetes Call-Center entweder über Anruf oder Erscheinen eines Patienten Impftermine vereinbart und bereits die erforderlichen Stammdaten angelegt werden. Hierbei wurde die Auslastung der einzelnen Impfstellen angezeigt und berücksichtigt. Wollte ein Patient einen Termin ändern bzw. absagen oder wollte der Patient einen Termin für eine seinerzeit noch ge- plante zweite Impfung vereinbaren, war dies unabhängig von der Impfstelle über die Anwendung problemlos möglich.

Im Empfangsbereich der jeweiligen Impfstelle konnte standardmäßig die Übersicht der Terminplanung eingesehen werden. Meldete sich nun ein Patient beim Empfang, konnte geprüft werden, ob dieser einen Termin hatte. Mit sehr wenigen Ausnahmen mussten Patienten, die überhaupt keinen Termin hatten, mit den Stammdaten neu angelegt werden. Darüber hinaus konnte im Empfang eine standardisierte personenbezogene Aufklärungsbescheinigung erstellt und ausgedruckt werden.

Die Erstellung dieser Impfdatenbank kostete das AfG €18649,68 (s. Tab. 1, Zeile 41, ebd.); der Erwerb von 2 Servern, davon einer mit separater Datenspeicherung für den externen Zugriff der Betriebsärzte, belief sich auf $€ 9313,33$ plus anteiligem VorOrt-Service für $1 \mathrm{Jahr}$ von $€ 246,66$ (Zeile 40, ebd.), die Installation des Webservers €2378,57 (Zeile 42, ebd.). Zusätzlich entfielen auf Beratungsleistungen für Vorarbeiten $€ 1713,60$ (Zeile 44, ebd.), auf die Schulung der Impfstellen $€ 4473,45$ (Zeile 43, ebd.) und für laufende Wartung und Betreuung der Datenbank (nur für 10 - 12/2009) €4688,12 (Zeile 45, ebd.). Für die Impfstellen des AfG wurden 9 Laptops (insgesamt $€ 11781,-$; Zeile 39, ebd.) beschafft. Insgesamt beliefen sich die dem AfG angefallenen, serverbezogenen Kosten (ohne Berücksichtigung von Abschreibungen) somit auf $€ 53244,41$.

\section{Aufbau einer Telefon-Hotline-Impfberatung}

Bereits ab dem 22.10.2009 wurde erneut eine Hotline („Bürgertelefon“) im AfG notwendig, die neben einer separaten telefonischen Impfanmeldestelle über die Impfmöglichkeiten und -risiken beraten sollte. Beide Telefondienste wurden mit nicht-ärztlichem Personal, letztere in Früh- und Spätschicht werktags zusätzlich noch mit bis zu 10 externen studentischen Hilfskräften besetzt; die Entwicklung der erforderlichen Schichten pro Einsatzwoche und die Anrufe pro Schicht sind in $\triangle$ Abb. 1 grafisch dargestellt. Ein Impfteam, innerhalb dessen Anamnese, Beratung und die Impfung selbst durchgeführt wurde, bestand in der Regel aus einer studentischen Hilfskraft zur Durchführung der Arzthelfertätigkeiten und einem Arzt. Die Impfungen fanden außerhalb der üblichen Dienstzeit statt (von 17-21 Uhr, in den ersten beiden Wochen auch samstags von 9-16 Uhr), um die Erreichbarkeit zunächst für die niedergelassenen Ärztinnen und Ärzte, Apotheken und deren Personal, später auch für möglichst viele Bürgerinnen und Bürger zu sichern. Aufgrund des zu erwartenden Impfandrangs nach der ersten Woche (26. 10. 2009 bis 1.11. 2009 noch 51 Erkrankte, in der darauf folgenden Woche bereits 207 Erkrankte, dann 827 Erkrankte) wurden bis zu 20 pensionierte Ärzte über die Landesärztekammer für die Impfteams zusätzlich stundenweise über Werkverträge beschäftigt. Ab dem 5. 10. 2009 wurde für sämtliche Beschäftigten des AfG eine Urlaubssperre ausgesprochen, was in einem Fall zu einer Übernahme der Stornokosten führte (€940,-).

Im Zeitraum vom 22.10.2009 bis 31.12.2009 entfielen für Bürgertelefon, Impfanmeldung und -durchführung sowie Logistik 


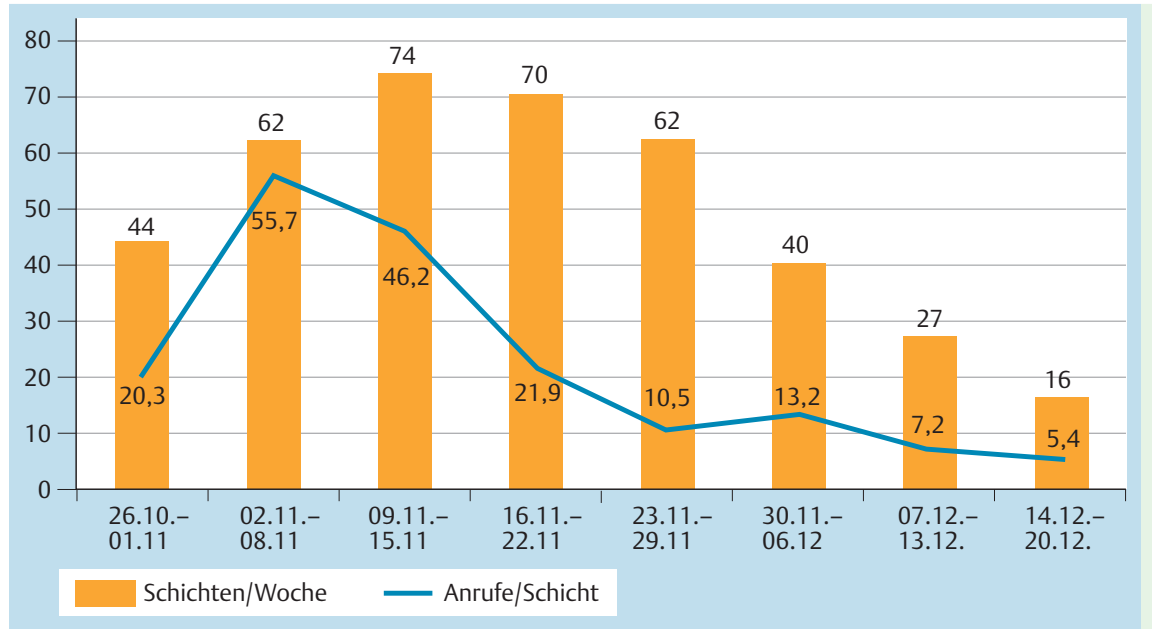

Abb. 1 Geleistete Dienste und durchschnittliche Anrufe in der Hotline pro Dienst und Mitarbeiter. Die Zahlen über den Balken zeigen die Summe der pro Woche in den Schichten eingesetzten Mitarbeiter; die Zahlen innerhalb der Balken dokumentieren die durchschnittliche Anzahl von Anrufen pro Schicht in der jeweiligen Woche.

\subsubsection{9 bis 31.12.2009 („Deutschlandphase“)}

\begin{tabular}{lrrr}
\hline PK* Bürgertelefon & Stunden & Kosten je Stunde & Kosten gesamt \\
\hline PK* Impfanmeldung & 758,20 & $49,00 €$ & $37151,80 €$ \\
\hline Überstunden ärztl. Personal & 362,70 & $49,00 €$ & $17772,30 €$ \\
\hline Überstunden Personal Verwaltung & 406,72 & $72,00 €$ & $29283,84 €$ \\
\hline PK* externe Ärzte & 283,61 & $60,00 €$ & $17016,60 €$ \\
\hline PK* externe Hilfskräfte/Studenten & 364,00 & $35,00 €$ & $12740,00 €$ \\
\hline abzgl. Erstattung aus Fondleistungen & 1620,00 & $15,00 €$ & $24300,00 €$ \\
(5€ pro Impfung bei 4281 Impfungen) & & & abzgl. 21305,00€ \\
\hline PK* gesamt & & & $116959,54 €$
\end{tabular}

* PK= Personalkosten
Tab. 3 Personalkosten für die „Deutschlandphase“ der H1N1Pandemie 2009.
$€ 138264,54$ an internen und externen Personalkosten (s. - Tab. 3 sowie Tab. 4, in der als Teilmenge von $\bullet$ Tab. 3 lediglich die Personalkosten für die Impfdurchführung dargestellt sind). Aufgrund des allmählich nachlassenden Impfinteresses wurde anschließend kein separates Impfteam mehr bereitgestellt und die Beantwortung von telefonischen Anfragen in der normalen Sprechstunde der Abteilung Infektiologie des AfG abgewickelt.

\section{Direkte Impfkosten}

Bei den im Folgenden dargestellten Kosten, werden nur direkte Kosten erfasst, die in unmittelbarem Zusammenhang mit der einzelnen Impfung stehen. Kosten für die Planung des Impfszenarios, die Logistik, die Einrichtung und den Aufbau der Impfstellen, sind nicht berücksichtigt. Die direkten Impfkosten setzen sich zusammen aus den für die Impfung entstandenen Personalkosten für die „Deutschlandphase“ der H1N1-Pandemie ( Tab.4a) sowie die dem AfG entstandenen Sachkosten ( $\bullet$ Tab. 4b). Die Personal- und Sachkosten pro Impfung, errechnet als Quotient der Summe von Personal- und Sachkosten und der Zahl durchgeführter Impfungen, sind in $\bullet$ Tab.4c) dargestellt.

Nachdem ab dem 22.10.2009 bereits eine Hotline („Bürgertelefon) eingerichtet war, wurden unter der Regie des AfG seit dem 26.10.2009 bis zum 31.3.2010 insgesamt 10375 Impfungen durchgeführt, hiervon 4261 Impfungen im Amt für Gesundheit und 6114 Impfungen durch Betriebsärzte Frankfurter Krankenhäuser (s. Abb. 2).

Die Personalkosten in Höhe von $€ 45018,84$ ( $\bullet$ Tab.4a) setzen sich zusammen aus Kosten für Überstunden von Ärzten des AfG, Werkvertragskosten für externe Ärzte sowie externe Studenten, wobei die vom Land Hessen aus dem Fonds für Impfleistungen gemäß Influenzaschutzimpfung-GKV-Leistungspflichtverordnung (ISchGKVLV) vom 19. August 2009 erstatteten $€ 5,-$ pro Impfung, d.h. von insgesamt $€ 21305$,- für die 4261 Impfungen, bereits abgezogen sind.

Da vom Hessischen Sozialministerium den Gesundheitsämtern lediglich Spritzen und Kanülen zum Impfstoff geliefert wurde, entstanden zusätzlich Sachkosten für weiteres Sanitätsmaterial (Pflaster, Desinfektionsmittel, Tupfer etc.), die von den Gesundheitsämtern selbst übernommen werden mussten. Hierfür fielen gemäß Auflistung ca. 8,9 Cent pro Impfung, für 4261 Impfungen insgesamt $€ 382,64$, an ( $\bullet$ Tab.4b). Kalkuliert man nun die Kosten pro Impfung ([€382,64 Sachkosten $+€ 45018,84$ Personalkosten]: 4261 Impfungen), so errechnen sich Einzelkosten von $€ 10,66$ pro Impfung exklusive des Impfstoffes ( $\bullet$ Tab.4c).

\section{Präventions- und Logistikkosten des Jahres 2009}

Obwohl die beschriebenen Personal- und Sachkosten sämtlich im Jahre 2009 anfielen, müssen zur Abbildung der für das laufende Jahr 2009 angefallenen Kosten mögliche Abschreibungen (AfA) für Sachkosten einbezogen werden. Tab. 5 zeigt, dass hiernach von den dauerhaften Anschaffungen im Jahre $2009 € 1156,85$ plus $€ 14150,83$, d.h. €15307,68 berücksichtigt werden können. Zusammen mit den laufenden, nicht abschreibungsfähigen Sachkosten von $€ 49211$, errechnet sich hieraus ein Gesamtsachkostenbetrag für das Jahr 2009 von $€ 64519,01$. Hiervon sind die direkten Sachkosten für die Impfung in Höhe von €382,64 (s. - Tab. 4.) noch abzuziehen, sodass €64136,37 verbleiben.

Die gesamten Präventionskosten des AfG im Hinblick auf die Eindämmung der A/H1N1-Influenza vor oder begleitend zur Impfaktion betragen im Jahre 2009 somit $€ 116959,54$ (Personalkosten „Deutschlandphase“, s. ๑ Tab.3) plus €21137,00 (Personal- 
Tab. 4 Direkte Personal- und Sachkosten des Amtes für Gesundheit für die Impfung.

\begin{tabular}{|c|c|c|c|}
\hline \multicolumn{4}{|l|}{ a) Personalkosten } \\
\hline \multicolumn{4}{|l|}{ 22. 10. 2009 bis 31.12. 2009 („Deutschlandphase“) } \\
\hline & Stunden & Kosten je Stunde & Kosten gesamt \\
\hline ärztliches Personal des Amtes (Überstunden) & 406,72 & $72,00 €$ & $29283,84 €$ \\
\hline externe Ärzte (Werkverträge) & 364,00 & $35,00 €$ & $12740,00 €$ \\
\hline externe Hilfskräfte/Studenten (Werkverträge) & 1620,00 & $15,00 €$ & $24300,00 €$ \\
\hline abzgl. $5 €$ Erstattung pro Impfung aus Fondleistungen (4261 Impfungen) & & & abzgl. 21305,00€ \\
\hline Personalkosten gesamt & 2390,72 & & $45018,84 €$ \\
\hline \multicolumn{4}{|l|}{ b) Sachkosten } \\
\hline Sachkosten & $0,0898 €$ je Impfung ${ }^{*}$ & & \\
\hline Anzahl der Impfungen & 4261 & & \\
\hline Sachkosten gesamt & $382,64 €$ & & \\
\hline \multicolumn{4}{|l|}{ c) Kosten für eine Impfung gegen A/H1N1 im Amt für Gesundheit } \\
\hline Personalkosten gesamt & $45018,84 €$ & & \\
\hline Sachkosten gesamt & $382,64 €$ & & \\
\hline Anzahl der Impfungen & 4261 & & \\
\hline Kosten pro Impfung & $10,66 €$ & & \\
\hline $\begin{array}{l}\text { Sachkosten gemäß realen Anschaffungskosten: } \\
1 \text { Pflaster } 0,0570 € \\
2 \text { Tupfer } 0,0140 € \\
2 \text { Desinfektionssprühstöße } 0,0188 € \\
\text { Summe } 0,0898 €\end{array}$ & & & \\
\hline
\end{tabular}

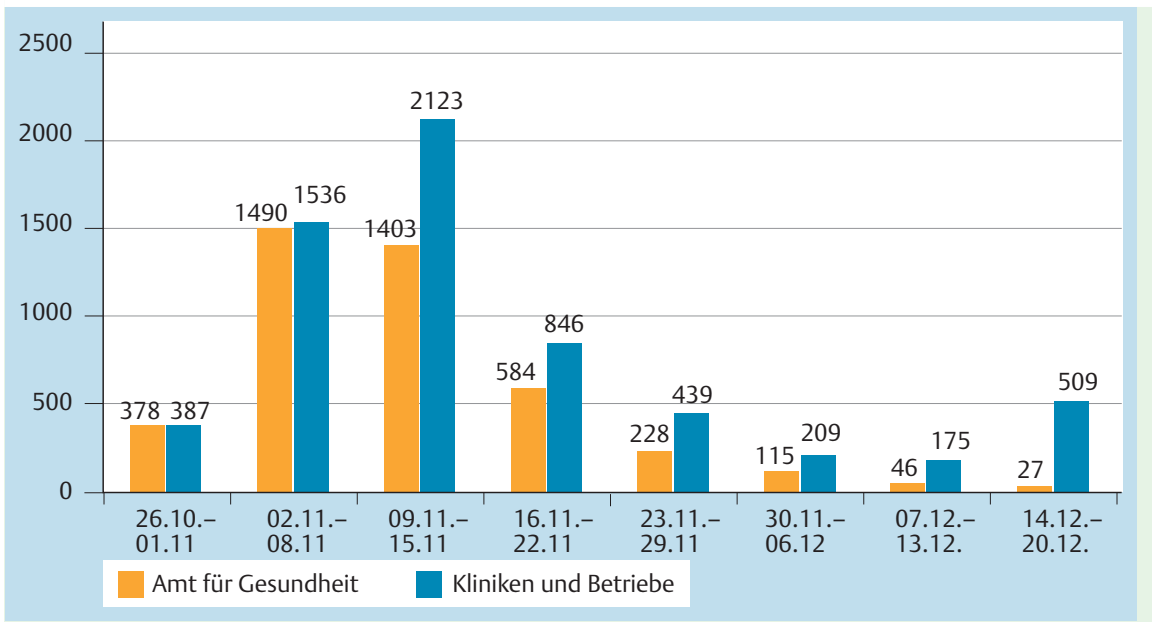

Abb.2 Zahl der wöchentlichen Impfungen im Amt für Gesundheit und in externen Impfstellen.

kosten „Mexikophase“, s. ๑ Tab.2) plus €64136,37 Sachkosten, insgesamt $€ 202232,91$. Bezogen auf die Bevölkerung der Stadt Frankfurt (664838, Stand 31.12.2008 [3]) betragen die Präventions- und Logistikkosten somit ca. €0,30 pro Person.

\section{Diskussion \\ $\nabla$}

Die Kosten der H1N1-Influenzapandemie im Jahre 2009 belasteten den Öffentlichen Gesundheitsdienst in Deutschland, trotz der vergleichsweise niedrigen Erkrankungszahl von 175603 Personen plus 53070 aggregierten Meldungen [4], in erheblichem Maße. Während in der Öffentlichkeit nach Ausrufung der postpandemischen Phase im Hinblick auf die Kosten der Bundesländer nur die Kosten für nicht verbrauchten Impfstoff beziffert werden [5], wurden die direkten monetären Aufwendungen des Öffentlichen Gesundheitsdienstes (ÖGD) bislang nicht analysiert. Die Strukturen des ÖGD wurden dabei in zweifacher Weise in Anspruch genommen: Nachdem das Potenzial der Pandemie nur schwer einzuschätzen war, galt in den ersten Wochen die Strategie der Eindämmung, mit dem Ziel, eine inländische Verbreitung von importierten A/H1N1-Infektionen möglichst lange zu vermeiden bzw. zu verlangsamen. Nachdem sich diese Strategie im Sommer als überholt erwies, galt es, Personen, bei denen im Fall einer Infektion ein erhöhtes Infektionsrisiko bestand, zu schützen und die Morbidität und Mortalität zu reduzieren [6]. Nach Entwicklung eines Impfstoffes wurde der ÖGD daher in die nationalen Impfstrategien eingebunden.

Unabhängig von der tatsächlichen Erkrankungszahl und der Impfbereitschaft in der Bevölkerung musste simultan eine kommunikative Plattform entwickelt werden, um federführend die Aufklärung der lokalen Bevölkerung über die neue Erkrankung und später die Impfstrategie sicherzustellen sowie die Logistik über eine rasche, kostensparende Massenimpfung zu gewährleisten und, je nach Bundesland in unterschiedlichem Ausmaß, aktiv an der Durchimpfung von Risikogruppen bzw. der Gesamtbevölkerung teilzunehmen. Hieraus ergibt sich zugleich eine begrenzte Aussagekraft der in dieser Analyse errechneten Kosten, da eine 
Tab.5 Sachkosten für das Jahr 2009 (Übertrag der Kostenauflistung in Tab. 1).

\begin{tabular}{|c|c|c|c|c|}
\hline & "Mexikophase“ & „Deutschlandphase“ & $\begin{array}{r}\text { Nutzungsdauer } \\
\text { in Jahren }\end{array}$ & $\begin{array}{l}\text { Abschreibung pro Jahr (= An- } \\
\text { schaffungskosten pro Jahr) }\end{array}$ \\
\hline \multicolumn{5}{|l|}{ Sachkosten: } \\
\hline laufende Sachkosten & $25681,81 €$ & $23529,52 €$ & & \\
\hline Kosten für dauerhafte Anschaffungen gesamt & $1156,85 €$ & $53456,33 €$ & & \\
\hline Schutzkappen für Fieberthermometer & $494,48 €$ & $0,00 €$ & & \\
\hline Kartei-Leitregister, Karteikästen & $180,42 €$ & $0,00 €$ & & \\
\hline Beistelltische & $0,00 €$ & $131,64 €$ & & \\
\hline Magnethaftschilder & $419,00 €$ & $0,00 €$ & & \\
\hline Fleecejacken, Strickhandschuhe, Arztkittel & $0,00 €$ & $370,41 €$ & & \\
\hline Messkolben & $0,00 €$ & $8,57 €$ & & \\
\hline Bode-X-Wipes-Eimer rot & $0,00 €$ & $416,20 €$ & & \\
\hline Transportwagen, Kühltasche, Ice-Akku & $0,00 €$ & $213,31 €$ & & \\
\hline Laptoptasche & $62,95 €$ & $0,00 €$ & & \\
\hline 10 Schlüsseltransponder & $0,00 €$ & $623,32 €$ & & \\
\hline Zwischensumme „dauerhafte Anschaffungen“: & $1156,85 €$ & $1763,45 €$ & & \\
\hline \multicolumn{5}{|l|}{ GWG $^{1}:$} \\
\hline $\begin{array}{l}\text { Wandsystem (separat nutzbare Trennwände } \\
\text { wegen Akustik in Call-Centern), Abdeckband }\end{array}$ & $0,00 €$ & $7610,04 €$ & 5 & $1522,01 €$ \\
\hline \multicolumn{5}{|l|}{$\mathrm{AV}^{2}:$} \\
\hline Latitude, N-Service Intel Core (9 Laptops) & $0,00 €$ & $11781,00 €$ & 3 & $3927,00 €$ \\
\hline $\begin{array}{l}2 \text { Server }(9.313,33 €)^{3} ; 4 \text { Jahre Vor-Ort-Service } \\
(986,65 €=246,66 € \text { anteilig für } 1 \text { Jahr })\end{array}$ & $0,00 €$ & $9559,99 €$ & 4 & $2390,00 €$ \\
\hline \multicolumn{5}{|l|}{ Imm.V } \\
\hline Erstellung einer Impfdatenbank & $0,00 €$ & $18649,68 €$ & 5 & $3729,94 €$ \\
\hline Installation eines Webservers & $0,00 €$ & $2378,57 €$ & 5 & $475,71 €$ \\
\hline Beratungsleistungen für Vorarbeiten & $0,00 €$ & $1713,60 €$ & 5 & $342,72 €$ \\
\hline $\begin{array}{l}\text { Zwischensumme } \\
\text { „dauerhafte Anschaffungen GWG, AV+ imm. V.“: }\end{array}$ & $0,00 €$ & $51692,88 €$ & & $12387,38 €$ \\
\hline Gesamtsumme „dauerhafte Anschaffungen“ & $1156,85 €$ & $53456,33 €$ & & \\
\hline $\begin{array}{l}\text { Kosten für dauerhafte Anschaffungen } \\
\text { (nach Berücksichtigung AfA) }\end{array}$ & $1156,85 €$ & $\begin{array}{r}14150,83 € \\
(12387,38 € \\
\text { plus } 1763,45 €)\end{array}$ & & \\
\hline
\end{tabular}

1 GWG (150€-1.000€) gem. gesetzlicher Bestimmungen ( 6 Abs. 2a Einkommenssteuergesetz - ESTG) grundsätzlich 5 Jahre Nutzungsdauer

${ }^{2}$ Erwerb von beweglichem Anlagevermögen. Nutzungsdauer It. Abschreibungstabelle der Stadt Frankfurt a. M.

${ }^{3}$ Nutzungsdauer It. Abschreibungstabelle der Stadt Frankfurt am Main 3 Jahre; durch den Servicevertrag erhöht auf 4 Jahre.

${ }^{4}$ Erwerb von immateriellem Anlagevermögen. Nutzungsdauer It. Abschreibungstabelle der Stadt Frankfurt a. M.

Übertragbarkeit der Ergebnisse für die Stadt Frankfurt am Main mit ihrer Zuständigkeit für den Rhein-Main-Flughafen auf das Bundesgebiet allenfalls eingeschränkt möglich ist.

Die Aufteilung der hierbei exemplarisch am Beispiel der Stadt Frankfurt am Main entstandenen Kosten für die Erfüllung beider zeitlich nachgeschalteten Strategiekonzepte zeigt, dass die direkten Gesamtkosten (Personal- und Sachkosten) für die durchgeführten Impfungen ( Tab.4a und $\bullet$ Tab.4b) mit insgesamt $€ 45401,48$ bzw. von $€ 10,66$ pro Impfung (exklusive der Kosten für den vom Land Hessen gestellten Impfstoff, - Tab.4c) einen kleineren Teil an den insgesamt durch die Pandemie für das Amt für Gesundheit entstehenden Kosten von €202232,91 ausmachen: Im Vordergrund stehen mit weiteren Kosten von $€ 156831,43$ der Personalbedarf für allgemeine Präventions- und Beratungsleistungen sowie Logistik und die Entwicklung eines modernen, servergestützten Systems, welches auch für andere impfpräventable Erkrankungsausbrüche in der Zukunft effektiv genutzt werden kann. Diese Entwicklung war notwendig geworden, da von der oberen Landesbehörde in der Anfangsphase der Vorbereitung auf die Impfkampagne keine Aussagen zur geplanten Impfstrategie gemacht werden konnten. Daher wurden in Frankfurt am Main drei verschiedene Strategien komplett durchgeplant und strukturell vorbereitet. Hierzu haben wir sehr frühzeitig eine Datenbank selbst entwickelt und von einer extern beauftragten Firma umsetzen lassen. Diese Datenbank war von vornherein so angelegt, dass sie allen Anforderungen gerecht werden wird: Sie sollte u.a. die komplette Fakturierung ermöglichen und alle zur Impfung gehörenden Informationen (Anmeldung der Patienten, Terminvergabe, durchgeführte Impfungen, evtl. Impfreaktionen etc.) mit erfassen. Zusätzlich sollte diese Datenbank jede Form der statistischen Abfrage ermöglichen und für alle anderen Szenarien im Sinne einer Pandemie einsetzbar sein.

Die für die Stadt Frankfurt am Main errechneten zusätzlichen Impfkosten von €10,66 pro Impfung sind im Vergleich zu den für die mit der Impfung verbundenen Dienstleistungen in der Influenzaschutzimpfung-GKV-Leistungspflichtverordnung (ISchGKVLV) vom 19. August 2009 erstatteten $€ 5$,- recht hoch. Entgegen der in einigen anderen Bundesländern durchgeführten Praxis einer Impfung durch niedergelassene Ärzte wurde vom zuständigen Hessischen Sozialministerium dennoch das Konzept einer durch den ÖGD gesteuerten Impfung in Hessen gewählt, da hierdurch von der kostengünstigsten Lösung ausgegangen werden konnte und gleichzeitig eine leichtere Kontrolle der $\mathrm{Zu}$ - und Abgänge des Impfstoffes möglich wurde.

Der erste bestätigte Erkrankungsfall mit der „Neuen Grippe“ trat in Frankfurt am Main relativ spät im Juni 2009 auf. Seitdem wurden mehr als 2200 bestätigte Fälle an diesem pandemischen 
Virus registriert. 5 Personen sind in Frankfurt am Main an der neuen Influenza A/H1N1 verstorben, darunter 2 Frankfurter Bürger.

In einer US-amerikanischen Studie wurde eine Dunkelziffer für jeden gemeldeten A/H1N1-Fall von 79:1, mit einer Spanne von $47-148$ berechnet [7]. Nimmt man als plausiblen Mittelwert 70 unentdeckte Fälle pro gemeldeten Influenzafall an, so ergeben sich für Frankfurt am Main insgesamt 155000 zumeist leicht infizierte Fälle, was $23 \%$ der Einwohner entspricht. Ob der immunologische Schutz dieser Patienten ausreichend ist, um in der nächsten Grippesaison noch gegen $\mathrm{A} / \mathrm{H} 1 \mathrm{~N} 1$ geschützt zu sein, ist gegenwärtig noch nicht beantwortet. Es könnte aber sein, dass ein Teil dieser Patienten weiterhin suszeptibel für dieses Virus ist und erneut erkranken könnte - eventuell sogar heftiger als beim ersten Mal. Dieses Phänomen wird, zusammen mit einer parallelen Gendrift, bei der spanischen Grippe für den katastrophalen Verlauf insbesondere der zweiten Welle 1918 diskutiert [8-10].

Zwangsläufig wird in diesen Zeiten die Notwendigkeit von Präventionsmaßnahmen durch den ÖGD immer wieder dramatisch steigen. Dies erfordert ungeachtet der besonderen Situation in Frankfurt am Main eine auskömmliche und flexible personelle und sachmittelbezogene Finanzierung des ÖGD, die im Hinblick auf die möglicherweise zu erwartenden infektiologischen Herausforderungen über die bislang vorherrschende Aufgabenstellung einer passiven Surveillance von Infektionskrankheiten weit hinausgehen dürften. In gleicher Weise betroffen sind auch andere medizinische Einrichtungen, insbesondere Krankenhäuser, bei denen durch den kürzlichen, unvorhersehbaren EHEC-0104/H4Ausbruch im Rahmen des geltenden DRG-Fallpauschalensystems erhebliche Kostendeckungslücken entstanden sind [11].

\section{Interessenkonflikt}

Die Autoren geben an, dass kein Interessenkonflikt besteht.

\section{Literatur}

1 Gaber W, Götsch U, Diel R et al. Screening for Infectious Diseases at International Airports: "The Frankfurt Model". Aviat Space Environ Med 2009; 80: 595-600

2 Wicker S, Rabenau HF, Bias H et al. Influenza A (H1N1) 2009: Impact on Frankfurt in due consideration of health care and public health. J Occup Med Toxicol 2010; 5: 10

3 Bürgeramt, Statistik und Wahlen. Bevölkerungsdaten der Stadt Frankfurt am Main 2011. http://www.frankfurt.de/sixcms/detail.php? id=2811\&_ffmpar[_id_inhalt] $=7524$

4 RKI. Ergänzung zum Epidemiologischen Bulletin Nr. 22 2010. http:// edoc.rki.de/documents/rki_fv/reU0lkuEjw5M/PDF/298YKR3HJIJ0lY. pdf

5 BMG. Gutachten zur Verbesserung der Wirtschaftlichkeit von Impfstoffen. Deutschland: 2010. http://www.bmg.bund.de/nn_1168248/ SharedDocs/Publikationen/DE

6 Bundesamt für Gesundheit. Grippe H1N1: Strategie nach der Pandemiewelle 2009 und Ausblick auf 2010. 2010. http://www.bag.admin. ch/influenza/01120/01134/index.html?lang=de

7 Reed C, Angulo FJ, Swerdlow DL et al. Estimates of the Prevalence of Pandemic (H1N1) 2009, United States, April-July 2009. Emerg Infect Dis 2009; 15: 2004-2007

8 Taubenberger JK, Reid AH, Janczewski TA et al. Integrating historical, clinical and molecular genetic data in order to explain the origin and virulence of the 1918 Spanish influenza virus. Philos Trans R Soc Lond B Biol Sci 2001; 29: 1829-1839

9 Taubenberger JK, Morens DM. 1918 Influenza: the mother of All Pandemics. Emerg Infect Dis2006: 15-22

10 Monsalvo AC, Batalle JP, Lopez MF. Severe pandemic 2009 H1N1 influenza disease due to pathogenic immune complexes. Nature Med 2011; 17: $195-199$

11 Bundesgesundheitsministerkonferenz. Finanzielle Konsequenzen der derzeitigen EHEC-Ausbrüche. Beschluss der 84. Gesundheitsministerkonferenz der Länder vom 30.6.2011, TOP 5.7. 